spektive unendlich und die Pflichten gegenüber künftigen Generationen gestalteten sich in einem ganz anderen Zusammenhang (Appukuttan Damodaran). In diesem Sinne meinte auch Medardo Tapia Uribe, dass nachhaltige Entwicklung als Prozess und nicht nur als Ziel zu verstehen sei. Dabei solle die Zivilgesellschaft mit ihren vielfältigen und komplexen Strukturen die zentrale Rolle spielen. Eine breite Partizipation aller Stimmen sei fundamental, wie er am Beispiel des Widerstandes der lokalen indigenen Bevölkerung in Mexiko gegen bestimmte Formen von Entwicklung und Wachstum zeigte. Caroline Robertson-von Trotha hob schließlich hervor, dass der kulturelle Pluralismus auf lokaler Ebene eine unverzichtbare Ressource sei, mit der noch nicht richtig umgegangen werde (,Kultur des kulturellen Pluralismus").

\section{Abschließende Bemerkungen}

Instruktiv war die Tatsache, dass sich die Teilnehmenden nicht von Beginn an auf eine Definition von Kultur festlegten. Insofern war im Sinne der Inter- und Transdisziplinarität ein aufschlussreiches Verfahren der Terminologisierung von „Kultur“ und „nachhaltiger Entwicklung“ zu beobachten. Alle Beteiligten waren sich einig, dass in einem nächsten Schritt die Erarbeitung einer methodologisch angemessenen Erfassung des Verhältnisses nachhaltiger Entwicklung und der kulturellen Dimension folgen solle. Die Erkenntnis, dass Nachhaltigkeit nur aus einer transdisziplinären Perspektive fokussiert werden kann, die nicht nur die Zusammenarbeit verschiedener wissenschaftlicher Disziplinen und Praxis impliziert, sondern vielmehr ein Umdenken im Verständnis von Wissenschaft selbst erfordert, stellt die größte Herausforderung dar. Diese kann nur in Form eines gemeinsamen Austauschs bewältigt werden, gleichzeitig zeigt sie aber auch die Notwendigkeit der wissenschaftlichen Ausgestaltung des Forschungsgegenstands.

\section{Perspektiven der sozialwissen- schaftlichen Technik- und Inno- vationsforschung}

Klausurtagung der Sektion Wissenschafts- und Technikforschung der Deutschen Gesellschaft für Soziologie

Stuttgart, 8.-9. Juli 2010

\section{von Ulrich Dolata, Jürgen Hampel, Felix Schra- pe und Stephanie Schulz, Universität Stuttgart}

Wohin könnte und sollte sich die sozialwissenschaftliche Technik- und Innovationsforschung in den kommenden Jahren bewegen? Welche theoretischen Themen und Ansätze, welche empirischen Untersuchungsgegenstände und welche methodischen Fragen sind besonders viel versprechend und zukunftsträchtig? Welche Impulse aus der internationalen Diskussion sollten in der deutschsprachigen Technik- und Innovationsforschung stärker aufgegriffen werden? Und welche Impulse könnte umgekehrt der deutschsprachige Diskurs stärker in die internationale Diskussion hineintragen?

Um diese Fragen kreisten die Diskussionen auf einer vom Lehrstuhl für Organisations- und Innovationssoziologie ausgerichteten Klausurtagung zu den „Perspektiven der sozialwissenschaftlichen Technik- und Innovationsforschung", die im Juli 2010 an der Universität Stuttgart stattfand. Die Tagung brachte 35 namhafte Wissenschaftlerinnen und Wissenschaftler zu einem intensiven Meinungsaustausch über den aktuellen Stand der Forschung und die künftigen Forschungsschwerpunkte des Fachs zusammen.

Nach einer ersten allgemeinen Debatte um die kontrovers diskutierte Frage, inwieweit der Innovationsbegriff auf soziale Zusammenhänge (soziale Innovationen) ausgeweitet werden sollte (Impulsstatements: Werner Rammert, TU Berlin, und Armin Grunwald, ITAS), wurden fünf Themenbereiche erörtert, in die jeweils durch kurze Impulsstatements eingeführt wurde:

- Veränderungen von Arbeitsprozessen und Alltagszusammenhängen durch neue Technologien (insbesondere durch das Internet), durch intelligente Infrastrukturtechnologien und durch hochtechnisierte Produktionssyste- 
me (Impulsstatement: Tanja Carstensen, TU Hamburg-Harburg);

- hybride Konstellationen, insbesondere neue Formen der Mensch-Maschine-Interaktion und die zunehmende Technisierung des Körpers (Impulsstatements: Johannes Weyer, TU Dortmund und Cornelius Schubert, TU Berlin);

- Internetforschung, die sich auf die Untersuchung neuer Formen sozialer Vergemeinschaftung konzentriert und Fragen der empirischen Erfassung und theoretischen Reflexion solcher Prozesse aufwirft (Impulsstatements: Christian Stegbauer, Universität Frankfurt a. M. und Niels Taubert, Universität Bielefeld);

- Formen und Verläufe soziotechnischen Wandels sowie mit neuen Technologien einhergehende strukturelle und institutionelle Veränderungen und Akteure, die diese Prozesse tragen (Impulsstatements: Hartmut HirschKreinsen, TU Dortmund und Stefan Böschen, Universität Bielefeld);

- Fragen der Gestaltung von Innovationsprozessen und deren Steuerungsmöglichkeiten durch neue Formen von Governance (Impulsstatements: Jan-Peter Voß, TU Berlin und Peter Wehling, LMU München).

\section{Technik, Arbeit, Alltag}

Anknüpfend an einen Schwerpunkt des Verbundes „Sozialwissenschaftliche Technikforschung“ aus den 1980er Jahren wurde ein klassisches Thema der Technik- und Arbeitssoziologie aufgegriffen: die Rolle, die Technik im Alltag sowie in Arbeitsprozessen zukommt.

Technische Systeme und vor allen Dingen das Internet spielen im Alltag eine immer wichtigere Rolle und definieren den Forschungsgegenstand „Technik und Alltag“ neu. Endgeräte wie Smartphones oder Notebooks und Social-MediaDienste wie „Facebook“ oder „Twitter" flexibilisieren sowohl die Kommunikation als auch die Informationsbeschaffung und fördern die Auflösung der ehemals klaren Grenzen zwischen Arbeit und Privatleben. Intelligente Technik dringt darüber hinaus vermehrt in die Haushalte ein. Dabei werden Energie- und Kommunikationstechniken neu verknüpft, vom ,intelligenten Stromzähler“ bis hin zum vollintegrierten „Smart Home“. Welche
Rolle diese Entwicklungen für die Veränderung des Alltags spielen, ist eine zentrale Fragestellung der sozialwissenschaftlichen Technikforschung.

Zudem tragen neue Technologien auch zum Wandel von Arbeitsprozessen bei. Zum einen schälen sich neue Formen professioneller und nichtbezahlter Arbeit im Internet heraus, die von der Produktion von Inhalten durch die Nutzer bis hin zur kommerziellen oder nicht-marktlichen Entwicklung technologischer Angebote im Rahmen von Entwicklergemeinschaften (z. B. in Open-Source-Projekten) reichen. Wie diese vielfältigen neuen Formen der Arbeit und Wertschöpfung im Netz strukturiert sind, welche Bedeutung interaktive Nutzerbeteiligungen an Innovationsprozessen („Open Innovation“), der Erstellung von Inhalten (,Open Content") oder der quelloffenen Programmierung („Open Source“) erlangen und in welchem Verhältnis sie zu klassischen Arbeitsformen stehen, wurden von den Teilnehmern als offene Fragen für künftige Forschungsvorhaben festgehalten. Neben der Untersuchung der neuen Formen der Arbeit im Internet sollte, darauf wurde auch hingewiesen, nicht übersehen werden, dass zum anderen auch die klassischen Formen der Industriearbeit einem Wandel unterliegen, insbesondere durch das Vordringen hochtechnisierter Systeme in der industriellen Produktion. Welche Auswirkungen der Umgang mit hochtechnisierten Produktionssystemen auf das Arbeitshandeln hat, ist im Vergleich zur Aufmerksamkeit, die dem Internet zuteilwird, bislang kaum untersucht worden und bildet eine wichtige Forschungsperspektive für die Arbeits- und Industriesoziologie.

\section{Hybride Konstellationen: Mensch-Maschine- Interaktion und Technisierung des Körpers}

Die Diskussion um Körper und Technik hat eine lange Tradition: Schon Heidegger hat darauf hingewiesen, dass das Hämmern nicht bereits durch das bloße Vorhandensein eines Hammers möglich werde, sondern erst eine entsprechende Körper-Technik in Kombination mit dem Hammer das Hämmern als Handlung ermögliche. Während der Hammer noch eindeutig als Werkzeug charakterisiert werden kann, entstehen heute hybride Konstellationen, in denen technischen Systemen Handlungsanteile zugeschrieben werden 
und so die Differenz zwischen menschlichem Körper und Technik verschwimmt.

Besonders fortgeschritten ist diese Hybridisierung etwa in modernen Flugzeugcockpits, in denen menschliche Handlungen sehr weitgehend um technische Systeme ergänzt werden, die nicht nur selbständig in die Steuerung eingreifen, sondern diese voll und ganz übernehmen. Fallstudien zeigen, dass diese Übernahme auch zu einer verteilten Handlungsträgerschaft führt: Die Piloten selbst räumen den Steuerungssystemen eigene Handlungsbeiträge ein. Ähnliches lässt sich etwa bei Fahrer-Assistenz-Systemen in modernen PKWs oder auf dem Finanzmarkt beobachten. Interessant für die weitere Forschung war die Diskussion um die Frage, auf welchen Ebenen sich hybride Konstellationen zwischen Mensch und Maschine aufspüren und analysieren lassen. Sie sind bislang vornehmlich auf der Mikroebene verteilten Handelns zwischen individuellen menschlichen und nicht-menschlichen Akteuren beobachtet worden. Das Beispiel der Finanzmarktkrise, deren abgründige Dynamiken wesentlich durch mitentscheidende weltweit vernetzte technische Systeme beschleunigt worden ist, zeigt, dass sich technisches Mithandeln auch als Makrophänomen analysieren lässt.

Auf der anderen Seite wurden unter den Stichworten „Human Enhancement“ und „Embodied Technology“ die Reichweiten und Auswirkungen direkter technischer Eingriffe in den menschlichen Körper erörtert. Zwar hat der Philosoph Arnold Gehlen schon in den 1950er Jahren den Menschen als Wesen bezeichnet, das durch Technik und Werkzeuge seine Mängel kompensieren müsse. Moderne Körper-Technologien aber gehen weit über einen Werkzeugcharakter hinaus und führen zu neuen Hybridformen des Handelns, die unter Umständen eine theoretische und methodologische Neujustierung des analytischen Instrumentariums notwendig werden lassen: Welche Rückwirkungen haben beispielsweise neue Reproduktionstechniken oder die Verlangsamung des Alterungsprozesses durch künstliche Organe auf die gesellschaftlichen Organisationsstrukturen? Da sich viele dieser Entwicklungen noch in einem Entwicklungsstadium befinden und sich soziale Veränderungen aller Vermutung nach erst auf lange Sicht bzw. graduell einstellen werden, wird es eine der vordringlichen Aufgaben der Soziologie sein, die individuellen und gesellschaftlichen Effekte dieser neuen Technologien zu verfolgen und ihre Konfliktpotenziale aufzuzeigen.

\section{Internetforschung}

Das Web steht als neues Technikfeld derzeit unter besonderer sozialwissenschaftlicher Beobachtung und bildete auch einen Schwerpunkt der Stuttgarter Diskussion. Besonders stark im Fokus der Aufmerksamkeit steht seit einigen Jahren die Untersuchung sozialer Vergemeinschaftungsprozesse im und durch das Web - v. a. im Rahmen von sozialen Netzwerken wie „Facebook“, von Blogs oder von „Wikipedia“. Im Zentrum stehen dabei v. a. die Beziehungen der User dieser Angebote zueinander sowie die dadurch entstehenden Relationen und Machtbalancen, die oft noch unverstanden sind und sowohl empirische wie auch theoretische und methodische Forschungsfragen aufwerfen.

Methodisch wurde ein Trend zur Netzwerkanalyse auf der Basis der relationalen Soziologie ausgemacht. Theoretisch wurde die Frage gestellt, ob sich die neuen Formen von Sozialität, die sich im Internet herausbilden, mit einer auf die OfflineWelt bezogenen Theoriesprache und ihren Struktur-, Akteurs- und Interaktionskonzepten noch angemessen abbilden lassen. Offen ist auch, wie und in welcher Weise Vergemeinschaftungsprozesse im Web restrukturierend auf soziale Kontexte zurückwirken, also welche Wechselwirkungen es zwischen der Offline- und der Online-Welt gibt. Das betrifft konkret auch das „Daten-Re-Entry“, also die Frage, wie und in welchem Ausmaß die im Web angehäuften Daten auf die soziale Sphäre zurückwirken, z. B. als Material in politischen Debatten (z. B. ,Wikileaks“), als Basis immer effektiverer und personenzentrierterer Werbung durch Internet-Unternehmen oder als frei verfügbarer Datenpool für wissenschaftliche Untersuchungen.

Kritisch wurde in diesem Zusammenhang angemerkt, dass sich die Analysen sozialer Netzwerke im Web bislang stark auf die unmittelbaren Beziehungen zwischen Usern konzentrieren, die kommerziellen Unternehmen als organisierende, strukturierende und kontrollierende Knoten in diesen Netzwerken dagegen weitgehend unbeachtet bleiben. Neue Internet-Plattformen 
wie „Facebook“ oder „StudiVZ“ werfen auch die Frage auf, inwieweit dadurch öffentliche Diskurse aus dem öffentlichen Raum in einen privatwirtschaftlich strukturierten Raum überwechseln und so der öffentliche Diskurs zum konstitutiven Teil eines Geschäftsmodells wird.

Schließlich wurde auch die Materialität des Netzes diskutiert und als eigenständiger Forschungsschwerpunkt definiert. Hinter den vielfältigen Web-Angeboten steht ja eine weiträumige Infrastruktur, die von großen Daten- und Rechenzentren mit großer regionaler Wirtschaftskraft und enormem Energieverbrauch getragen werden. Damit geraten die Analyse der Soft- und Hardware-Entwicklung im Kontext solcher Datenzentren, deren wirtschaftliche Bedeutung für die Regionen, in denen sie angesiedelt werden (und die mit ihrem Betrieb einhergehenden ökologischen Auswirkungen), in den Blick.

\section{Soziotechnischer Wandel und Gover- nance von Innovationsprozessen}

In den letzten beiden Themenblöcken wurde intensiv darüber diskutiert, wie unterschiedliche gesellschaftliche Ebenen in Innovationsprozessen zusammenwirken, wie Prozesse soziotechnischen Wandels verlaufen und auf welche Weise sich dieser Wandel gestalten lässt. Untersuchungen zu den Wechselwirkungen von Innovationsprozessen und sozialen Dynamiken gehören schon lange zum Kerngeschäft sozialwissenschaftlicher Technik- und Innovationsforschung. Die Stuttgarter Tagung konnte diesem Thema wichtige neue Forschungsimpulse geben.

Zum einen wurde von Workshopteilnehmern darauf verwiesen, dass das Zusammenspiel regionaler, sektoraler und nationaler Innovationssysteme nach wie vor ungeklärt ist, und diskutiert, welche institutionellen Komplementaritäten und Konflikte zwischen den verschiedenen Ebenen relevant sind. Zweitens schiebt sich neben die Fragen nach den sozialen Grundlagen und institutionellen Rahmenbedingungen von Technikentstehungs- und Innovationsprozessen, die etwa im Kontext der Technikgeneseforschung, des Innovationssystem-Ansatzes oder des „Varieties-of-Capitalism“-Konzepts ausgiebig diskutiert worden sind, zunehmend die komplementäre Perspektive, die hinterfragt, welchen Beitrag neue Technologien selbst zum System- und Institutionenwandel leisten. Mit diesem Perspektivwechsel wird die Frage aufgeworfen, welche organisationalen, institutionellen und strukturellen Veränderungen mit dem Aufkommen neuer technologischer Möglichkeiten einhergehen und wie sich ein solcher technikinduzierter Wandel konzeptionell fassen lässt.

Drittens wurde angeregt, den Verlauf derartiger Prozesse soziotechnischen Wandels als graduelle Transformation zu untersuchen, für die typisch ist, dass sie sich nicht in Form einmaliger und radikaler Brüche, sondern als Resultat einer Vielzahl technischer, organisationaler, struktureller und institutioneller Veränderungen Bahn brechen und über längere Zeit hinziehen. In diesem Kontext wurde auch als Forschungsfrage formuliert, wann und unter welchen Bedingungen derartige Wandlungsprozesse wieder in stabile Strukturen münden und ab welchem Schwellenwert technikvermittelte graduelle Wandlungsprozesse in substanziell neue Strukturen umschlagen.

Mit Blick auf die in Technisierungsprozesse involvierten Akteure wurde dafür plädiert, den Blick stärker als bislang auch auf solche Akteure zu richten, die sich nicht mit klassischen Organisationsbegriffen fassen lassen. Konkret können das technikskeptische Bürger sein, die neue Technologien mehrheitlich und stabil nicht (oder nur eingeschränkt) akzeptieren (wie z. B. die Grüne Gentechnik). Das können auch eigenwillige und selektierende Konsumenten neuer technischer Angebote sein, die sich diese anders als erwartet aneignen (z. B. bei neuen kommunikationstechnischen Produkten). Und das können schließlich unkonventionelle Technikentwickler und -nutzer sein, die massenhaft, ohne kommerzielle Absichten und außerhalb bestehender Marktstrukturen mit neuen technologischen Möglichkeiten zu spielen beginnen - und damit zur De-Kommodifizierung von Produkten und zur Erosion bestehender Märkte beitragen können (z. B. Software-Communities und File Sharer von digitaler Musik oder von Filmen).

Die Untersuchung dieser Akteure wirft eine Reihe interessanter und innovativer Forschungsfragen auf: Welche Rollen können sie in Prozessen technologischen, organisationalen und insti- 
tutionellen Wandels spielen? Wie beeinflussen sie Innovationsprozesse und welches Innovationspotenzial haben sie? Oder auf welche Weise können sie Gewichtsverschiebungen zwischen Formen marktlichen und nicht-marktlichen Tauschs anstoßen?

Im Themenfeld „Governance von Innovationsprozessen“, das insgesamt stark vom Konzept des ,transition management" geprägt wird, wurden zum einen neue Formen der Innovationspolitik vorgestellt, die nicht nur eine quantitative Beschleunigung von Innovationstätigkeit bzw. den nicht weiter qualifizierten „Markterfolg“ Maßstab nimmt, sondern gezielt Innovationsprozesse befördern kann, die zur Bewältigung gesellschaftlicher Herausforderungen beitragen. Und zum anderen wurde die antizipierende diskursive Governance diskutiert, die darauf zielt, Technikkonflikte zu verhindern, wie sie etwa hinsichtlich der Gentechnik aufgetreten sind. Ähnliche Konflikte könnten zukünftig z. B. auch um die Nanotechnologie oder die synthetischen Technologie entstehen. Dabei bestand bei den Teilnehmern Konsens darüber, dass bei der Betrachtung solcher Governance-Prozesse das gesamte Spektrum möglicher Akteure einbezogen werden sollte: Auch zivilgesellschaftliche Akteure, die kaum oder nur schwach organisiert sind, könnten einen beachtlichen Einfluss auf die Technikentwicklung ausüben. Theoretisch lässt sich hier Anschluss an analytische Ansätze finden, die sich mit „Promising Technology" beschäftigen: Sie gehen davon aus, dass nicht nur die Sachtechnik selbst relevant ist für die Technikentwicklung, sondern auch die mit ihr diskursiv verknüpften Visionen, Erwartungen und Bilder.

Bemerkenswert an den Governance-Diskussionen um war zum einen, dass sich rund um die „new modes of governance“ ein zum Teil beachtlicher Steuerungsoptimismus breit gemacht hat, der innovativen Gestaltungskonzepten eine Wirkmächtigkeit zuschreibt, die empirisch erst noch zu bestätigen ist. Darüber hinaus wurde angemerkt, dass bei der Untersuchung neuer Governance-Formen klassische Ausprägungen technikpolitischer Aushandlung (etwa im Rahmen klientelistischer, korporatistischer oder themenzentrierter Netzwerke), die keineswegs obsolet sind, sowie Einfluss- und Machtasymmetrien zwischen den beteiligten Akteuren nicht ausgeblendet werden dürfen.

\section{Ausblick}

Insgesamt war dies eine sehr produktive Klausurtagung, die eine ganze Reihe interessanter neuer Forschungsfragen und -themen aufgeworfen hat. Das lag nicht nur an den Diskussionen selbst, sondern auch an der Anlage der Veranstaltung: Die Themenschwerpunkte waren nicht vorgegeben, sondern haben sich aus den von den Teilnehmerinnen und Teilnehmern eingereichten Abstracts ergeben. Anders als bei thematisch fokussierten Tagungen ist es darüber hinaus gelungen, Wissenschaftlerinnen und Wissenschaftler aus unterschiedlichen disziplinären und thematischen Zusammenhängen zusammenzubringen, die im normalen Gang der Dinge vergleichsweise wenig miteinander zu tun haben. Am Ende der Tagung standen erste Vorschläge für vertiefende Veranstaltungen, für Kooperationen und für zu initiierende Forschungsschwerpunkte. 